Revista de Psicología de la PUCP. Vol. XX, 2, 2002

\title{
Peruanas y españolas que consumen drogas: un estudio desde la perspectiva del género ${ }^{1}$
}

\author{
Milton Rojas Valero' \\ CEDRO - Lima \\ Nuria Romo Avilés ${ }^{3}$ \\ Universidad de Granada
}

\begin{abstract}
Se examinan dos realidades distintas de consumo de drogas desde la perspectiva del género. Los datos epidemiológicos y algunos patrones de consumo en mujeres peruanas y españolas son analizados estableciendo semejanzas y diferencias. Se pretende mostrar la diversidad que se esconde tras el concepto "mujer drogodependiente", que afecta a nacionalidades, clases sociales, etnias y otras variables sociales. En el análisis del caso peruano se analizan datos procedentes de las encuestas epidemiológicas disponibles, de estudios de dependencia y de los derivados de las personas atendidas en el centro Lugar de Escucha en Lima. En el caso español, se presentan datos procedentes de las encuestas epidemiológicas realizadas a nivel nacional y de la información obtenida de la investigación sobre mujeres consumidoras de drogas de síntesis.

Palabras clave: drogas, consumo, género, mujer, Perú, España.
\end{abstract}

\section{Peruvian and Spanish women using drugs: A study under the gender perspective}

This article examines two different realities concerning the use of drugs under the gender perspective. Epidemiological information and some patterns of use are analyzed establishing differences and similarities. The objective is to show the diversity behind the concept "women in drug consumption", a phenomena that affects nationalities, social classes and other social issues. In the case of Peru we analyze data gathered through epidemiological surveys, studies of use-dependence and of people contacted throughout the Lugar de Escucha service (telephone line for drug addicts) in Lima. In Spain, we analyze two sources: the epidemiological studies made at national level and a research about women using chemical drugs.

Key words: drugs use, gender, woman, Peru, Spain.

Agradecemos a Carmen Meneses, profesora de la Universidad Pontificia de Comillas en Madrid, por la revisión de este artículo.

Licenciado en psicología clínica de la Universidad Ricardo Palma. Ha realizado estudios de maestría en fármaco-dependencia en la Universidad Peruana Cayetano Heredia. Trabaja en el Centro de Información y Educación para la Prevención del Abuso de Drogas (CEDRO) y se desempeña como supervisor del servicio para consumidores de drogas $\mathrm{Lu}$ gar de Escucha (Lima). Dirección: Av. Roca y Bologna No. 275, Lima 18, Perú. Correo electrónico: mrojas@cedro.org.pe. 



\section{Peruanas y españolas: iiguales y diferentes en el uso de drogas?}

La influencia del género en las distintas formas de uso de drogas ha sido con frecuencia olvidada en los estudios sobre drogodependencias. La diferente realidad social que afecta a las mujeres peruanas y españolas no cambia esta premisa. Cuando las mujeres usan drogas, incumplen los roles que les han sido asignados socialmente sin que influyan en esto los continentes de residencia, lo que lleva a un menor interés por estudiar los motivos específicos que las hacen usar drogas y las consecuencias que de ello se derivan.

En Sudamérica, particularmente en el Perú, y en Europa, especialmente en España, los estudios de género que se han acercado a la investigación del consumo de drogas por las mujeres han tenido un insuficiente desarrollo histórico. Dentro de los límites de los datos obtenidos, las investigaciones han sido desarrolladas para responder a algunas preocupaciones o preguntas como derechos y salud sexual y reproductiva, el desarrollo social asociado a efectos de la violencia, el maltrato doméstico y el abuso de alcohol y otras drogas en algunos trabajos descriptivos (ej. en el Perú: Dador, 1998; Vargas, 1993; Yánez, 1996).

Sin embargo, en los últimos años, las investigaciones que incluyen la perspectiva de género están cobrando importancia en la literatura científica que aborda los distintos usos de drogas. La relevancia que está adquiriendo el papel que desempeña la mujer en las socieda-

Doctora en antropología social por la Universidad de Granada. Es profesora asociada del Departamento de Antropología y Trabajo Social de la mencionada universidad. Dirección: C/ Rector López Arguieta, s/n. Granada. España. Correo electrónico: nromo@ugr.es. 
des latinoamericanas y occidentales actuales ha contribuido en este sentido. La mirada a la problemática que presenta la población femenina y su influencia en la dinámica de las diferentes estructuras sociales está cambiando y permitiendo la aparición de nuevos trabajos de investigación que cubren ámbitos relacionados con el uso de drogas por las mujeres (ej. en España: Orte, 1998; 1998; Meneses, 2001 y en el Perú: Rojas, 1998, 1996; Vigo, 1999 y Molla y Raguz, 1997)

Partimos de que el género es un sistema de relaciones sociales, simbólicas y psíquicas en las que se sitúa de forma diferente y casi siempre desfavorable a las mujeres. María Luz Esteban propone la definición de Lourdes Benería y define el género como el conjunto de creencias, rasgos personales, actitudes sentimientos, valores, conductas y actividades que diferencian a varones y mujeres a través de un proceso de construcción social (Esteban, 2001). El género permite así contemplar los sexos como entidades sociales, políticas y culturales, superando las limitaciones del concepto de sexo, que parece referirse a algo natural, primario, esencial y aparentemente sometido a escasas transformaciones históricas (Ortiz Gómez, 1997). La aplicación de la perspectiva de género a la investigación social permite visualizar las desigualdades sociales entre varones y mujeres, y la intervención sobre ellas.

La desigualdad de género afecta la forma en que las mujeres viven su salud y cómo ésta es vista socialmente. Los usos de drogas por las mujeres están influenciados por la forma en que ellas viven sus roles de género y por cómo son contempladas en sociedad cuando consumen drogas.

La información disponible muestra que los varones son los principales usuarios de drogas ilegales. Sin embargo, parece que las mujeres están destinadas a tratar sus malestares con tranquilizantes. La "tranquilidad recetada" de la que hablaba la argentina Mabel Burín se produce en países que difieren en aspectos culturales, sociales y 
económicos como son Perú y España superando cualquier tipo de diferencia.

Las mujeres en España y en otros países europeos están accediendo a mejores situaciones que otras generaciones previas en el sistema educativo, en el trabajo y en la participación en entornos públicos y de ocio tradicionalmente ocupados por los varones. En los últimos años se viene constatando en los estudios epidemiológicos una mayor prevalencia de consumo entre las adolescentes que se aproximan e igualan a los varones en sus formas de consumir drogas y que han reducido la brecha entre los sexos, mostrándose esta realidad en las encuestas realizadas a población adulta y población escolar. Entre las nuevas formas de uso de drogas, destaca su proximidad a los varones en uso de sustancias como las drogas de síntesis.

En el Perú se están conociendo y registrando usos incipientes de drogas como las de síntesis, pero la mayor accesibilidad y menor precio de los derivados de coca llevan a un perfil de mujer usuaria de drogas distinto, con mayor abuso y prevalencia de problemáticas derivadas del consumo de derivados de la hoja de coca.

A pesar de las deficiencias de los estudios epidemiológicos en el Perú y del contraste entre los tipos de usuarias de drogas más conocidas, comparar y exponer los datos disponibles en los dos países nos permite aproximarnos a una realidad que quizás necesite de una mejor aplicación de la perspectiva de género y nuevos horizontes metodológicos para comprender mejor similitudes y diferencias entre las mujeres que usan drogas en contextos económicos y culturales distintos.

Bajo el termino mujer drogodependiente se esconde una realidad con múltiples mujeres diferenciadas por sus países de origen, su etnia, clase social y otras variables sociales que deberá ser estudiada en sus distintas facetas igual que lo ha sido el varón drogodependiente. 
Éste es un estudio exploratorio en el que hemos tratado de hacer un ejercicio de reflexión conjunta para analizar de forma descriptiva y comparada la realidad de dos países cercanos y distantes en la vivencia del uso de drogas por las mujeres, planteándonos los siguientes objetivos:

1. Describir la situación de las mujeres con relación a los distintos usos de drogas en España y el Perú, analizando las diferencias y similitudes entre los sexos en estos dos contextos sociales.

2. Analizar las pautas de uso de drogas que más inciden en las mujeres, comparando la problemática asociada en los dos países. Esto permitirá, en el futuro, proponer modos comparativos de intervención y análisis que permitan el diseño de programas de salud pública en ambos países.

\section{Epidemiología del uso de sustancias psicoactivas en el Perú en base al sexo}

En el Perú, el uso del alcohol, entre otras sustancias como la hoja de coca y los alucinógenos, se inscribe dentro de los contextos socio-culturales del país. Posiblemente, desde la fase pre-hispánica son los varones quienes han consumido mayoritariamente tanto las drogas denominadas lícitas como las ilícitas (Domic, 1980).

En la actualidad, el uso y abuso de alcohol y otras drogas capaces de generar adicción varía de acuerdo al sexo de la persona que las usa, siendo el consumo mayor en el caso de los varones con relación a todas las drogas. En el caso de los tranquilizantes se observa que el porcentaje de Prevalencia de $\mathrm{Vida}^{4}$ (PV) es similar en ambos sexos $(2.5 \%$ y $2.4 \%$, respectivamente). De esta manera, se confirma

$4 \quad$ Prevalencia de Vida (PV). En los estudios epidemiológicos que evalúan los patrones de uso de sustancias, la PV de uso de una sustancias a lo largo de la vida del entrevistado se mide a través de una sola pregunta o de un conjunto de preguntas por sustancias que interrogan al entrevistado respecto de si "ha usado alguna vez" la sustancia en cuestión. 
Peruanas y españolas que consumen drogas

que la PV en las mujeres peruanas urbanas es alto, tal como se puede observar en el Cuadro 1.

\section{Cuadro 1}

Porcentaje de la prevalencia del consumo de drogas según sexo

\begin{tabular}{|lcc|}
\hline Droga & $\begin{array}{c}\text { Varones } \\
\%\end{array}$ & $\begin{array}{c}\text { Mujeres } \\
\%\end{array}$ \\
\hline Tabaco & 59.9 & 31.1 \\
Alcohol & 83.4 & 76.4 \\
Cannabis & 1.2 & 0.2 \\
PBC & 1.2 & 0.0 \\
Cocaína & 0.9 & 0.0 \\
Inhalables & 0.5 & 0.2 \\
Tranquilizantes & 2.5 & 2.4 \\
Otras drogas & 2.1 & 3.2 \\
\hline
\end{tabular}

Nota. Fuente: CONTRADROGAS ${ }^{5}, 1999$.

Retomando el Cuadro 1, en cuanto a la PV de vida del uso de alcohol, llama la atención la alta tasa en ambos sexos $(83.4 \%$ en los varones y $76.4 \%$ en las mujeres). En e' caso de las mujeres en particular, pareciera confirmar la tendencia a acortarse la brecha que las separa de los varones. Si consideramos los estudios epidemiológicos peruanos previos, se observa una curva ligeramente ascendente en cuanto a la PV en las mujeres, mientras que en los varones se observa un patrón más estable en el consumo de alcohol ( ${ }^{6}$ Ferrando, 1990; Rojas y Ferrando, 1993; Rojas y Castro de la Mata, 1997; Jutkowitz, Arellano, Castro de la Mata, Davis, Elison, Jerí, Shaycoft y Timana, 1987; Rojas y Castro de la Mata, 1999).

5 CONTRADROGAS, es la Comisión de Lucha Contra el Consumo de Drogas, oficina interlocutora del gobierno peruano para lo referido al tema de las drogas. Este estudio epidemiológico se desarrolló en 1998. La muestra incluyó a 4,831 personas de zonas urbanas de la costa, sierra, selva y Lima Metropolitana.

CEDRO, es el Centro de Información y Educación para la Prevención del Abuso de Drogas con sede en la ciudad de Lima, Perú. Es un Organismo No Gubernamental creado en 1986, el mismo que ha desarrollado seis estudios epidemiológicos nacionales en ciudades urbanas de las tres regiones del Perú. 
En cuanto al tabaco, encontramos que los patrones de la PV en ambos sexos se inscribe en una línea más estable al compararlos con los resultados de investigaciones anteriores (Ferrando, 1990; Ferrando y Rojas, 1993; Rojas y Castro de la Mata, 1997; Jutkowitz, Arellano, Castro de la Mata, Davis, Elison, Jerí, Shaycoft y Timana, 1987; Rojas y Castro de la Mata, 1999). Este fenómeno puede obedecer a variados factores. Uno de ellos podría ser las campañas de prevención sistemáticas, la ausencia de anuncios publicitarios en los medios de comunicación durante el día así como la promulgación de nuevas leyes antitabáquicas ${ }^{7}$.

Varones y mujeres tienen patrones distintos de consumo de drogas ilegales (Figura 1). En el caso de los varones adolescentes, la probabilidad de consumo de drogas ilegales, según CONTRADROGAS (1998), es casi diez veces mayor entre los de 14 a 16 años que entre los de 12 a 13 años. El 13.2\% de los varones y el 1.5\% de las mujeres han consumido drogas ilegales alguna vez en su vida.

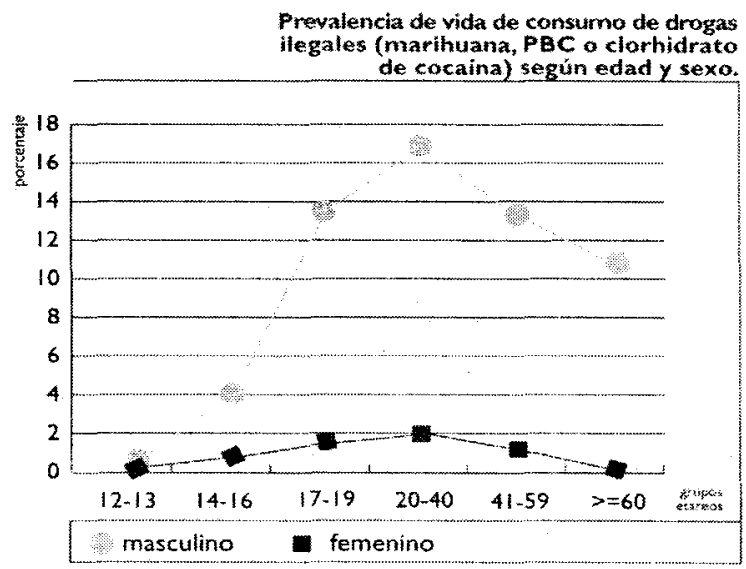

Figura 1. Prevalencia de vida de consumo de drogas ilegales (Marihuana, PBC o clorhidrato de cocaína) según edad y sexo. Fuente: CONTRADROGAS (1999).

7 En este sentido ha resultado de especial relevancia la promulgación de la Ley Antitabáquica No. 25357 de 1998. 
Peruanas y españolas que consumen drogas

En cuanto a las distintas consecuencias que pueden derivarse del uso de drogas, uno de ellas la dependencia, el Cuadro 2 nos presenta la dependencia según los criterios de la Décima Clasificación Internacional de Enfermedades versión ICD-10. Estos datos muestran cómo los varones adictos al alcohol y al tabaco, son casi cuatro veces más que las mujeres.

Respecto a las drogas ilegales, incluyendo las sustancias inhalantes, el grueso de los dependientes son varones. En las mujeres, la tendencia más destacada es su mayor dependencia a los tranquilizantes cuando las comparamos con los varones $(0.4 \%$ en ellas y $0.3 \%$ en ellos).

\section{Cuadro 2}

Personas dependientes del consumo de drogas en el Perú según sexo ${ }^{8}$

\begin{tabular}{|lrrrr|}
\hline Tipo de droga & \multicolumn{2}{c}{ Varones } & \multicolumn{2}{c|}{ Mujeres } \\
& \multicolumn{1}{c}{$n$} & $\%$ & \multicolumn{1}{c}{$n$} & $\%$ \\
\hline Alcohol & 749770 & 16.0 & 262798 & 4.9 \\
Tabaco & 577883 & 12.4 & 238086 & 4.4 \\
Marihuana & 2514 & 0.1 & 684 & 0.01 \\
Pasta Básica de Cocaína & 27700 & 0.6 & 00 & 0.0 \\
(PBC) & & & & \\
Clorhidrato de Cocaína & 3844 & 0.1 & 684 & 0.01 \\
Inhalantes & 4499 & 0.1 & 798 & 0.01 \\
Tranquilizantes & 14288 & 0.3 & 19318 & 0.4 \\
\hline
\end{tabular}

Nota. Fuente: CONTRADROGAS (1999).

Respecto al uso de cannabis, cocaína o inhalantes, las mujeres son poco dependientes y representan una baja tasa del total $(0.01 \%)$. En el Cuadro 2, llama la atención la ausencia de datos para el caso

8 En esta oportunidad CONTRADROGAS asume los criterios clínicos de la Décima Clasificación Internacional de Enfermedades versión (ICD-10) para evaluar la severidad del consumo y la dependencia a sustancias, comprendiendo neuroadaptación, tolerancia, abstinencia y pérdida del auto-control. 
de dependencia de la PBC en mujeres, aunque sí encontramos un $0.01 \%$ de adictas a la cocaína.

Esta escasa prevalencia en el consumo y dependencia a drogas como la PBC entre las mujeres, teniendo como base los estudios epidemiológicos, contrasta con los datos derivados de la atención que solicitan cuando sienten que tienen un problema con alguna sustancia psicoactiva. En el Cuadro 3 se observa que el $18.54 \%$ de la demanda de ayuda es del sexo femenino. Las drogas relacionadas por los usuarios con "problemas" y por las que se pide ayuda en Lugar de Escucha son la marihuana (4.8\%), alcohol (2.7\%), cocaína (2.3\%) y el de los psicofármacos con el $2.1 \%$, PBC $1.4 \%$, éxtasis $0.7 \%$, tabaco $0.1 \%$; no se encuentran casos de mujeres que hayan solicitado ayuda por tener problemas con la heroína, pero sí se observa $0.24 \%$ (4 casos) de consumo de heroína en los varones.

\section{Cuadro 3}

Consumidores/as de drogas atendidos en Lugar de Escucha (Lima, Perú) en el 2001, según sexo

\begin{tabular}{|lcrrrrr|}
\hline Motivo de consulta & \multicolumn{2}{c}{ Mujeres } & \multicolumn{2}{c}{ Varones } & \multicolumn{2}{c|}{ Total } \\
& \multicolumn{1}{c}{$f$} & \multicolumn{1}{c}{$\%$} & \multicolumn{1}{c}{$f$} & \multicolumn{1}{c}{$\%$} & \multicolumn{1}{c|}{$\%$} \\
\hline Marihuana & 82 & 4.84 & 371 & 21.90 & 453 & 26.74 \\
Alcohol & 46 & 2.72 & 258 & 15.23 & 304 & 17.95 \\
Cocaína & 40 & 2.36 & 278 & 16.41 & 318 & 18.77 \\
Psicofármacos & 37 & 2.18 & 40 & 2.36 & 77 & 4.55 \\
PBC "Tabacazos" & 25 & 1.48 & 192 & 11.33 & 217 & 12.81 \\
Éxtasis & 12 & 0.71 & 17 & 1.00 & 29 & 1.71 \\
Tabaco & 2 & 0.12 & 13 & 0.77 & 15 & 0.89 \\
Ludopatía & 2 & 0.12 & 19 & 1.12 & 21 & 1.24 \\
Heroína & - & 0 & 4 & 0.24 & 4 & 0.24 \\
Otros & 68 & 4.01 & 188 & 11.10 & 256 & 15.11 \\
\hline Total & 314 & 18.54 & 1,380 & 81.46 & 1,694 & 100.00 \\
\hline
\end{tabular}

Nota. Fuente: Casuística Anual 2001 del Programa Lugar de Escucha de CEDRO (Lima). 
Queda claro que el principal motivo de atención en Lugar de Escucha es el que se relaciona con el consumo de marihuana y otros derivados cannábicos (26.74\%), luego se encuentran las consultas por problemas relacionados con el alcohol y la cocaína $(17.95 \%$ y $18.77 \%$, respectivamente).

Respecto a las diferencias entre los varones y las mujeres que piden atención en este servicio suponen el $81.46 \%$ y $18.54 \%$ del total respectivamente. Es decir, de cada diez personas que son atendidas en el servicio, dos son mujeres.

Si comparamos estos datos con los encontrados en 1989 (Rojas, 1989) en el mismo servicio fueron atendidos un $11 \%$ de Mujeres y $89 \%$ de Varones. Estos son datos correspondientes al primer año de funcionamiento del servicio Lugar de Escucha. Si los comparamos con los obtenidos en el 2001, se evidencia que el porcentaje de mujeres atendidas se ha duplicado.

La visibilidad de las mujeres en centros como Lugar de Escucha contradice su invisibilidad en los datos derivados de los estudios epidemiológicos que hemos analizado y que muestran una muy baja o nula prevalencia de consumo de algunas drogas como la cocaína o la $\mathrm{PBC}$ entre las mujeres.

Una de las explicaciones a esta situación puede estar en los problemas de los estudios epidemiológicos, realizados a través de encuestas en hogares, en cuanto a la validez, confiabilidad y otras limitaciones para registrar comportamientos que, como el uso de drogas, son encubiertos y clandestinos. El mayor control social sobre determinadas conductas de riesgo como las asociadas a los usos de drogas, que generalmente contradicen los roles femeninos asignados socialmente, puede hacer que las mujeres declaren menos en las encuestas. Asimismo, su relación con lo que se ha denominado "feminización de la pobreza" puede que lleve a que las usuarias de dro- 
gas sean encontradas con menor frecuencia en domicilios en los que son entrevistadas y por lo tanto, al igual que en otros aspectos de la vida social, sean aquí también sobrepresentadas.

\section{Perfil de la mujer consumidora de cocaína fumada $\left(\mathrm{PBC}^{\text {" }}\right)$ en el Perú}

En el Perú no se habían realizado investigaciones que indagaran sobre características clínicas, psicosociales, culturales y familiares de mujeres que abusan o son adictas a drogas como la PBC hasta 1996. CEDRO desarrolló una investigación exploratorio-descriptiva en 65 adictas de PBC según los criterios clínicos del DSM III-R de la dependencia a sustancias. La muestra de estudio se clasificó en dos grupos: A: jóvenes comprendidas entre 14 y 22 años; y, B: adultas comprendidas entre 23 y 41 años (Rojas, 1996).

El perfil social de las mujeres consumidoras de cocaína fumada o PBC, correspondía con una mujer de aproximadamente 25.6 años de edad y con una situación socio-económica que las situaba en los niveles medio y bajo de la sociedad peruana. Gran parte de las mujeres de esta muestra estaban desempleadas o inactivas y habían completado los ciclos educativos primarios o secundarios.

El patrón de uso de PBC de estas mujeres está caracterizado por $1 \mathrm{a}^{10}$ compulsión. En esta población, la dosis promedio es de 65

y Pasta Básica de Cocaína (PBC) o cocaína fumada (Colombia: bazuco, Bolivia: pitillo, Chile: pasta base). Para Castro de la Mata (1988), se trata de una mezcla de diversas sustancias que se produce durante el proceso de extracción de los alcaloides de las hojas de coca. El componente principal es la cocaína que, en la sustancia no adulterada, debe representar más del $80 \%$ del total en su masa. Los otros componentes son productos de descomposición de la cocaína como la ecgonina y la benzoilecgonina, otros alcaloides como la tropacocaína y algunas sales como los sulfatos o carbonatos. Su reacción es alcalina. Su consumo genera rápidamente adicción, con un marcado comportamiento antisocial, deterioro físico y psíquico (Navarro, 1992: Nizama, 1991 y Rojas, 1999)

10 La compulsión generada por el fumado de la PBC está caracterizada por un marcado nivel de apetencia o "hambre" de droga, el mismo que hace renovar reiterativamente la do- 
Peruanas y españolas que consumen drogas

11 "tabacazos" (cigarrillos de PBC y tabaco) por sesión de consumo, que suele prolongarse durante 8 horas. En la muestra de estudio también se encuentra un tiempo promedio de consumo de 5 años, con un tiempo máximo de abstinencia de 5 meses. La modalidad de consumo no suele estar asociada a la ingesta de bebidas alcohólicas (consumo "a secas"), siendo la calle o los denominados" "fumaderos", el lugar de preferencia para el consumo en solitario o con un grupo pequeño de otros consumidores y consumidoras.

En lo que respecta a los argumentos que suelen esgrimir estas mujeres para justificar la continuación del consumo de la cocaína fumada, la mayoría aludía a "problemas personales". Las observaciones clínicas muestran algunas enfermedades relacionadas con su dependencia entre las que destacan: desnutrición, caries dental o enfermedades venéreas. Por otro lado, se determinaron los siguientes signos y síntomas: adelgazamiento, agresividad, depresión (correlacionado con intentos de suicidio), midriasis y anemia.

En relación al mundo sexual de estas mujeres, la edad de inicio de la actividad sexual suele ser a los 15 años. El 69.23\% de estas mujeres admitieron que existe asociación entre el consumo de la cocaína fumada y la prostitución.

En la estructura familiar de estas mujeres se encuentran sistemas variados (completa, incompleta y desintegrada). Respecto a la carga familiar, el promedio de hijos en la muestra es 1. Dentro del sistema

sis del consumo. De esa forma, el sujeto puede llegar a fumar un número importante de cigarrillos de PBC por sesión de consumo de 6 a 8 horas, con breves intervalos de descanso, pudiendo llegar hasta a días de consumo.

1 El "Tabacazo" es el cigarrillo compuesto por la mezcla entre PBC y cigarrillo, el mismo que es elaborado artesanalmente por el mismo adicto. Para asegurar una buena mezcla entre la droga, el tabaco y la combustión, insertan palitos de fósforos quebrados.

12 Los "fumaderos" son espacios abiertos o casas no. necesariamente abandonados y otros inmuebles donde se concentran los adictos de PBC para fumar la droga. Se trata de zonas de difícil acceso, donde suelen frecuentar adictos, micro-comercializadores y delincuentes. 
familiar se encuentra la presencia de por lo menos un hermano adicto, igual que en el caso de la cocaína fumada.

\section{Perfil de la mujer consumidora de cocaína (aspirada) en el Perú}

Para la determinación del perfil de la mujer consumidora de cocaína o clorhidrato de cocaína, CEDRO desarrolló una investigación descriptivo-retrospectiva con una muestra de 627 casos de mujeres, las mismas que fueron abordadas telefónicamente en primera instancia para posteriormente ser atendidas en los consultorios en términos de evaluación y diagnóstico psicológico y médico psiquiátrico, así como de consejo y motivación (Rojas, 1999).

De esta población, se determinó el diagnóstico de dependencia a la cocaína en el $13.20 \%$ ( 83 casos), dentro de patrones de policonsumo de drogas. En contraposición con las consumidoras de cocaína fumada, las usuarias de cocaína aspirada se inscriben en estratos socio-económicos medio, medio alto y alto. El promedio de edad es de 25,6 años. Estas características pueden obedecer a varios factores, como el costo de la sustancia y ciertos estereotipos que giran en torno al consumo de esta modalidad de cocaína y que la asocian con personas de mejor situación económica y social.

El precio ${ }^{13}$ del gramo de cocaína en el Perú ha tenido una drástica reducción en estos últimos años. El precio va a depender de la calidad de la sustancia, la modalidad de adquisición y sitio de consumo. Parece ser que ello a originado que el consumo de cocaína se halla extendido a otros estratos sociales peor situados, entre otros factores.

13 Hacia marzo del 2002, en Lima y Callao los precios de 1 gramo de cocaína pueden variar de 4 a 8 dólares. Un "Kete" de PBC (envoltura de papel con PBC) cuyo peso promedio es $0,3 \mathrm{~g}$, puede costar entre 0.09 centavos y 0.15 centavos de dólar. En ambos casos, va a depender de la pureza, lugar de venta, modalidad de compra, entre otros factores. 
En cuanto al grado de instrucción de la muestra estudiada, se encuentra que mayoritariamente son mujeres con secundaria completa e instrucción superior (completa o incompleta). De esta manera, este grupo se diferencia de la población de consumidoras de cocaína fumada, donde se encontró un nivel educativo de secundaria incompleta y primaria.

Estas son mujeres que están empleadas aún considerando que se trata de trabajos no estables; encontrándose otra diferencia respecto a las usuarias de cocaína fumada, donde mayoritariamente están desempleadas. Las actividades laborales encontradas son: Agente vendedor, secretaria, modelo, profesora, entre otras ocupaciones.

Respecto al estado de salud de estas mujeres, destacamos que es común encontrar rinitis crónicas, perforación del séptum nasal y colapso del tabique nasal. En cuanto a las observaciones obstétricas, hay abortos espontáneos y partos prematuros. En lo cardiovascular, hipertensión arterial e infarto al miocardio. En lo neurológico se hallan estados de pánico, hipertensión arterial, hemorragias intracerebrales y subaracnoideas. En la esfera psico-social y conductual, resalta la agresividad, déficit motivacional, promiscuidad e intentos de suicidio.

En el acercamiento a la personalidad antes de la iniciación del consumo de cocaína, se registra: depresión mayor, desorden ciclotímico, desordenes: bipolar (maniaco-depresiva), hiperactivo, comportamental y de personalidad.

El perfil indica que hay un consumo con una frecuencia interdiaria, con una dosis que oscila entre 2.5 a 3.5 grs. por sesión de 8 horas de consumo. El tiempo promedio de consumo al momento de la investigación es de 6 años. El consumo de la droga no está asociado mayoritariamente a la ingesta de bebidas alcohólicas y se lleva a cabo preferentemente en la casa, discotecas, bares y hoteles, sola o con un grupo reducido de personas. 
Una gran parte de estas mujeres se iniciaron en la actividad sexual a los 14 años. Su uso de cocaína suele estar asociado a la promiscuidad y la prostitución, siendo frecuente que hayan sufrido algún aborto.

En el área familiar se encuentra bastante similitud con las consumidoras de cocaína fumada o PBC, como quiera que se encuentran estructuras familiares variadas (completa, incompleta y desintegrada). En este contexto, se puede encontrar consumo de drogas en el entorno inmediato de la consumidora (esposo o pareja, amigos, padre, hermano).

\section{El consumo de sustancias psicoactivas en España: datos de las encuestas epidemiológicas en base al sexo}

En España, los datos derivados de las encuestas epidemiológicas o de registros de tratamiento muestran como tendencia general que las drogas ilícitas suelen usarse en mayor proporción por los varones que por las mujeres. Entre las de comercio legal, los varones consumen algunas con más intensidad, por ejemplo, tabaco o alcohol en algunos grupos de edad ${ }^{14}$. En general, la información derivada de estas encuestas indica que las mujeres consumen más drogas de prescripción médica que los varones y que suelen usar menos drogas, tanto de las comercializadas legalmente como de las ilegales ${ }^{15}$.

Los sedantes e hipnóticos son de las drogas más consumidas entre las mujeres españolas según las encuestas analizadas. Los datos

14 Es posible que estos datos se vieran matizados por las diferencias entre la prevalencia en las distintas regiones o en las distintas clases sociales, detalles que no hemos podido alcanzar aquí sobre la base de los datos disponibles.

15 Para el análisis de las prevalencias de uso de drogas hemos utilizado los resultados derivados de las dos principales encuestas sobre consumo de drogas ilegales en el ámbito nacional con las que contamos en España desde 1994. En concreto, hemos utilizado dos encuestas del Plan Nacional sobre Drogas: Encuesta Domiciliaria de Población Adulta para mayores de 15 años en todo el territorio español, 1996 y la Encuesta sobre Drogas de la Población Escolar, 1997. 
de la encuesta domiciliaria sobre uso de drogas muestran que un $12.5 \%$ de la población estudiada había consumido tranquilizantes durante los 12 meses previos al momento de ser encuestados y un $8 \%$ hipnóticos (DGPND, 1996).

Las mujeres eran las principales consumidoras de estas drogas en ambos casos: el $16.8 \%$ y $10.6 \%$ de mujeres las habían consumido durante el año anterior a ser encuestadas, frente a un $7.9 \%$ y un $5.3 \%$ de varones. Del mismo modo, los medicamentos son más usados por las adolescentes españolas cuando las comparamos con los chicos. En la Encuesta sobre Drogas a la Población Escolar (DGPND, 1997), la proporción de consumidores de tranquilizantes o pastillas para dormir sin prescripción médica era bastante más alta entre las chicas que entre los chicos. Un $5.6 \%$ de las chicas y un $3.1 \%$ de los chicos habían consumido estas sustancias en los doce meses previos a la encuesta. Estos datos muestran cómo las diferencias de género en torno al consumo de estas sustancias en España y su mayor incidencia entre las mujeres, se inician con la adolescencia y se mantienen en la madurez.

La evolución del consumo de tabaco, en particular entre las mujeres, es un campo de interés en los últimos años en los distintos ámbitos de estudio de las drogodependencias. Más del $50 \%$ de la población adulta entrevistada en la Encuesta Domiciliaria de población mayor de 15 años ha fumado en alguna ocasión. En el momento de ser encuestados fumaban un $36.1 \%$ de los varones y mujeres entrevistados, de los que un $30.4 \%$ lo hacían a diario. Los porcentajes más altos de fumadores diarios se dan entre los varones. Un $39.3 \%$ de este tipo de fumadores son varones, frente a un $22.1 \%$ de mujeres (DGPND, 1996).

Respecto al uso de alcohol, las mujeres suelen beber con menor intensidad que los varones. Los patrones de uso que relacionan a las mujeres con la embriaguez siguen caracterizándose por la falta de ex- 
ceso, ya que los varones son los que más beben a diario con una ratio que va del $30 \%$ de los varones al $7.5 \%$ de las mujeres. Los bebedores con riesgo alcohólico ${ }^{16}$ son en mayor medida los varones que se encuentran entre 40 y 54 años que toman en los días laborables y también los varones de edades más jóvenes que toman los fines de semana $^{17}$ (DGPND, 1996).

En la Encuesta Escolar se muestra un cierto cambio de tendencia y el aumento del consumo de ambas sustancias, tabaco y alcohol, entre las chicas. La proporción de las chicas que fuman en las edades que cubre la encuesta es mayor que la de los chicos. El número de consumidores de alcohol adolescentes, especialmente aquellos que beben diariamente los fines de semana, son similares en su distribución en cuanto al sexo.

La proporción de chicas que había fumado tabaco durante los 30 días previos a la encuesta fue de un $34.5 \%$ frente a un $23.7 \%$ de los chicos. A pesar de este mayor consumo entre las féminas, hay que destacar que la intensidad en el consumo parece ser menor entre ellas. El número medio de cigarrillos fumados diariamente por las chicas fue de 7 frente a 8.4 que fumaron los chicos (DGPND, 1997).

A pesar de que en la Encuesta Escolar de 1996 no se encontraran diferencias en la proporción de consumidores de bebidas alcohólicas según el sexo, los chicos bebían de forma más intensa que las chicas.

Estos datos indican que la cautela, definida como el control ante los usos más intensivos, es el rasgo más característico de la relación

16 El riesgo alcohólico se mide por la cantidad de c.c. de alcohol puro bebida al día. En este estudio se establece en + de 50 c.c. diarios.

17 En los grupos considerados en las encuestas realizadas por el Comisionado para la Droga de la Junta de Andalucía como de 'bebedores abusivos', es decir, los que beben a niveles altos, excesivos o de gran riesgo, se situaban el $2.4 \%$ de las mujeres entrevistadas en 1996 , frente al $13.5 \%$ de los varones que se encontraban en esos grupos de bebedores el mismo año. 
de las mujeres con el tabaco y el alcohol, a pesar de que en ambas sustancias las mujeres se van aproximando a los varones en las prevalencias de consumo. Sin embargo, los patrones de uso más intensivos se siguen produciendo entre los varones. Podríamos decir que los riesgos que implican los usos intensivos diferencian a los sexos.

Después del tabaco, el alcohol y los psicofármacos, el cannabis es la droga más consumida en España. En base a los resultados de la Encuesta de Población General realizada por el Plan Nacional sobre Drogas, la habían usado alguna vez en la vida, un $11.3 \%$ de los varones y mujeres entrevistados; un 5.8\% en los últimos 12 meses y el $27.2 \%$ en el último mes. Los varones que han probado la sustancia alguna vez en la vida duplican a las mujeres y cuatro varones por cada mujer la han consumido en los treinta días antes de ser entrevistados (Cuadro 4) (DGPND, 1996).

\section{Cuadro 4}

Frecuencia de consumo de cannabis en mayores de 15 años (España)

\begin{tabular}{|ccc|}
\hline$f$ & Mujeres & Varones \\
\hline Alguna vez & 7.2 & 15.6 \\
Último año & 3.3 & 8.4 \\
Último mes & 1.1 & 4.3 \\
\hline
\end{tabular}

Nota. Fuente: Elaboración a partir de los datos de DGPND/EDIS 1996.

Del mismo modo, el cannabis es la sustancia más consumida entre los adolescentes españoles tras el tabaco y el alcohol. En los 30 días antes de ser entrevistados, declaraban haber consumido cannabis un $15.7 \%$. La proporción sobre la base del sexo estaba en $25.9 \%$ de chicos por $20.7 \%$ de chicas que habían consumido la sustancia (DGPND, 1997). Al igual que ocurre con el tabaco y el alcohol, las diferencias de sexo se reducen, por lo tanto, entre los adolescentes si los comparamos con la población general. 
En cuanto a los usos de drogas más problemáticos, como los de cocaína o heroína, los datos de la encuesta domiciliaria de 1996 indican que más de dos varones por cada mujer habían consumido cocaína durante el último año antes de ser entrevistados. Del mismo modo, los varones vuelven a duplicar a las mujeres cuando se les preguntaba por su consumo de esta sustancia durante el mes previo a su entrevista (Cuadros 5 y 6 ).

\section{Cuadro 5}

Frecuencia de consumo de cocaína en mayores de 15 años (España)

\begin{tabular}{|ccc|}
\hline$f$ & Mujeres & Varones \\
\hline Alguna vez & 1.7 & 4.1 \\
Último año & 0.8 & 2.2 \\
Último mes & 0.2 & 0.5 \\
\hline
\end{tabular}

Nota. Fuente: Elaboración propia a partir de los datos de la DGPND/EDIS 1996.

En la población escolar, un $2.6 \%$ del total de los entrevistados había usado cocaína en el mismo período de tiempo. Durante los 30 días antes de realizarse la encuesta, habían usado cocaína el $1.5 \%$ de los adolescentes entrevistados. La ratio entre varones y mujeres descendía de nuevo en la población escolar cuando comparamos sus resultados con las encuestas a la población general, siendo el $2 \%$ de las mujeres las que la habían usado, frente al $3.3 \%$ de los varones.

En cuanto al consumo de heroína, los datos referidos a la población general española, muestran que entre las personas que han usado alguna vez en la vida esta sustancia los varones triplican a las mujeres y casi se mantienen en la misma proporción las mujeres y varones que la han consumido en el último mes. (Un 1,2\% de los varones la había consumido alguna vez en la vida frente a un $0,4 \%$ de las mujeres y un $0,5 \%$ de los varones en el último mes frente al $0,2 \%$ de las mujeres) (DGPND, 1996). 
Peruanas y españolas que consumen drogas

Cuadro 6

Frecuencia de consumo de heroína en mayores de 15 años (España)

\begin{tabular}{|ccc|}
\hline$f$ & Varones & Mujeres \\
\hline Alguna vez & 1.2 & 0.4 \\
Último año & 0.7 & 0.3 \\
Último mes & 0.5 & 0.2 \\
\hline
\end{tabular}

Nota. Fuente: Elaboración propia a partir de los datos de la DGPND/EDIS 1996.

La información proporcionada por las encuestas epidemiológicas muestra como los usos problemáticos de drogas son llevados a cabo en España fundamentalmente por varones. Las diferencias de sexo parecen reducirse en las edades adolescentes pero se marcan definitivamente en la madurez, quizás cuando las mujeres asumen sus roles principales, los de madres y esposas. Desde la privacidad y bajo la presión social, es difícil mantener conductas rechazas socialmente como las relacionadas con el consumo de drogas.

Cuando las mujeres se acercan a los varones en la prevalencia de uso de algunas drogas como tabaco o alcohol, la cautela es el rasgo que las describe por consumir con menos intensidad y evitar conductas de riesgo al intensificar los consumos.

\section{Nuevos usos y perfiles: la mujer consumidora de drogas de sínte- sis en España}

Los usos de drogas de síntesis entre los que se incluye el éxtasis, han constituido la novedad más destacada respecto a los distintos usos de drogas en España en la última década. Las usuarias de este tipo de drogas han desbancado en España al perfil típico de drogadicta representado por la usuaria compulsiva de heroína y problemática en los distintos aspectos de sus vidas. 
El término drogas de síntesis ${ }^{18}$ aglutina a un grupo de sustancias, cuyo consumo se extiende en Europa a finales de los años ochenta usadas, principalmente, en contextos recreativos asociados a la cultura juvenil de la fiesta o "rave". De ellas, las más frecuentemente utilizadas han sido las feniletilaminas. Dentro de este grupo de sustancias, la más conocida es el éxtasis. Este nombre suele utilizarse para designar a la MDMA (3-4 metilendioxi-metanfetamina) (Roig Traver, 1990). Las consumidoras de drogas de síntesis suelen ser policonsumidoras de un amplio grupo de sustancias entre las que se encuentra la cocaína, el cannabis o el LSD.

El consumo de estas sustancias de modo recreativo y asociadas a la cultura juvenil de la fiesta o "rave" se extiende desde finales de los años ochenta en los distintos países europeos (Gamella y Álvarez Roldán, 1999, 1999; Gamella, Álvarez Roldán y Romo, 1997a). Su popularidad se asocia a la emergencia de un nuevo estilo musical conocido como acid house o tecno, forma de música de baile electrónica rápida, y a la organización de grandes fiestas o "raves": celebraciones musicales que se extienden a lo largo del fin de semana y que tienen en los D-J o "pinchadiscos" sus figuras centrales (EMCDDA, 1997) ${ }^{19}$.

En España, el consumo de MDMA y sus análogos ha pasado por distintos períodos en los que la intensidad y popularización de su consumo ha ido variando. La encuesta domiciliaria realizada en España en 1999 por la Dirección General del Plan Nacional sobre Drogas estimaba que un $0.2 \%$ de los encuestados habían consumido éxtasis en los 30 días anteriores a la encuesta con estas características:

18 Este termino ha sido reconocido como el más apropiado en comparación con el de "drogas de diseño" tras la recomendación realizada por Gamella y Roldán, 1997. Su uso ha sido aceptado por otros profesionales españoles (Por ejemplo, véase, Mas et al. 1998; Elzo y Vielva, 1998) como más adecuado para designar este grupo de sustancias.

19 La historia internacional del movimiento ha sido estudiada por Saunders, N., 1995 o Collin, M., 1998 entre otros. 
la mayoría varones ( 7 de cada 10), de entre 20 y 24 años de edad, seguidos de los jóvenes de 15-19 años. La mayoría con estudios de FP/ BUP, seguidos en importancia por los universitarios/as y la mayoría solteros/as (DGPND, 1999).

Los datos de la última encuesta escolar realizada por el Observatorio Español sobre Drogas muestran que la diferencia en la incidencia del consumo entre varones y mujeres es menor en los grupos más jóvenes. Según la Encuesta Escolar del 2000, la prevalencia de consumo de éxtasis entre estudiantes de secundaria según sexo estaba en un $1.6 \%$ de mujeres de la población que había consumido esta droga frente a un $3.4 \%$ de varones. Un $4.6 \%$ de las chicas las había consumido alguna vez en la vida frente a un $6.8 \%$ de los varones. Es decir, las mujeres se acercan más a los varones en los índices de experimentación con la sustancia, pero aumentan sus diferencias con los varones cuando miramos al consumo que realizan en el último mes y año (Cuadro 7).

\section{Cuadro 7}

Prevalencia de consumo de éxtasis en estudiantes de secundaria (14-18 años) según sexo (España, 2000)

\begin{tabular}{|ccc|}
\hline$f$ & Varones & Mujeres \\
\hline Alguna vez & 6.8 & 4.6 \\
Último año & 5.9 & 3.7 \\
Último mes & 3.4 & 1.6 \\
\hline
\end{tabular}

Nota. Fuente: Encuesta sobre drogas a la población escolar. 2000. DGPNSD. Observatorio Español sobre Drogas.

Todos estos datos esconden el perfil de unas mujeres que se acercan a los varones en sus escenas de ocio. En la imagen pública las usuarias de drogas han sido identificadas como usuarias de heroína, con una fuerte problemática asociada a sus usos de drogas que las han llevado a padecer consecuencias sanitarias como las enfermedades de transmisión sexual, entre las que ha destacado el VIH/SIDA y 
a llevar a cabo conductas reprobadas socialmente como la prostitución o el abandono de los hijos.

Las mujeres que usan drogas de síntesis, entre las que destaca el éxtasis, consumen drogas en el ámbito de lo recreativo y normalizado en la visión del 'otro'. En lo referido a la esfera privada, son mujeres que consumen y han consumido drogas sin ser madres ni esposas, sin padecer el miedo a perder a sus hijos e hijas o parejas. Su consumo de sustancias psicoactivas como el de las drogas de síntesis se mantiene de manera más integrada socialmente que el que se produce en las mujeres usuarias de heroína retratadas en otros estudios como los de Rosenbaum, Taylor o Meneses en España.

Las mujeres se han incorporado a las nuevas formas de usos de drogas que se extienden a finales de los años ochenta en entornos poco criminalizados y más normalizados. El nuevo contex to de uso de drogas se ha unido a una mejor situación de las mujeres en España, incorporadas progresivamente a la esfera pública, con mejores niveles educativos que los varones y mayores márgenes de permisividad para participar en pautas de ocio que sólo unas generaciones antes eran impensables.

Esta integración social de la mujer que consume drogas de síntesis no elimina las necesidades de atención que empiezan a percibirse en los servicios sanitarios españoles. Si bien es cierto que no han llegado a provocar un problema de salud pública como el producido por los usuarios/as de heroína, pero los distintos problemas relacionados con los usos que se hacen de las drogas de síntesis en fiestas y las conductas de riesgo asociadas requieren intervenciones destinadas a la reducción de riesgos entre este tipo de usuarias de drogas. 
Peruanas y españolas que consumen drogas

\section{Reflexiones finales}

- El consumo de drogas está determinado en gran parte por el contexto sociocultural donde se produce. La tradición histórica o la oferta, junto a otros factores, llevan a patrones de consumo de drogas distintos en ambos países.

- Los roles de género influencian y determinan el uso que hacen las mujeres de las drogas, presentando realidades similares en contextos económicos y culturales muy distintos. Este es el caso de los psicofármacos de tipo benzodiazepinas y analgésicos que son usados de manera predominante por las mujeres en el Perú y España.

- En este sentido, destacamos que los datos disponibles en el Perú muestran deficiencias y levantan dudas que manifiestan la necesidad de estudios que utilicen otras metodologías de investigación que no sean sólo las epidemiológicas. Por ejemplo, hemos observado que aparecen diferencias entre los datos de prevalencia y las necesidades de ayuda y atención entre las mujeres que usan drogas como la cocaína o PBC. Asimismo, en el caso de los tranquilizantes, mujeres y varones son similares en prevalencia, pero son más las mujeres que piden atención por problemas relacionados con el consumo de estas sustancias.

- En el Perú, tras los psicofármacos, destacan los usos de los derivados cannábicos, cocaína aspirada y fumada. Del mismo modo, se encuentra que el consumo de cocaína fumada (PBC) en contraste con la cocaína aspirada, difieren en cuanto a las áreas demográfica, laboral y educacional; encontrándose similitudes en las áreas de la salud, clínico, psico-sexual, familiar y psico-social.

- Los estudios epidemiológicos sobre el consumo de drogas legales como el alcohol muestran que las mujeres han ido creciendo 
en número como usuarias y acortando la brecha que las separaba de los varones. En los próximos años veremos si esta tendencia se estabiliza.

- En España, la novedad más destacada son las drogas de síntesis. En el Perú, el consumo de este tipo de drogas en las mujeres no aparece en los estudios epidemiológicos, aunque sí en las casuísticas clínicas en un nivel incipiente. La mejora en la posición social de mujeres en España y su mayor grado de acceso e integración en las pautas de ocio de los varones las ha llevado a incorporarse con fuerza a sus pautas de uso de drogas. Este hecho se observa en las menores diferencias entre varones y mujeres en las encuestas escolares cuando las comparamos con las destinadas al estudio de la población general.

- La ausencia de diferencias en el consumo de algunas drogas entre los sexos parece estabilizarse en la vida adulta. Es en la edad adulta cuando las mujeres españolas se posicionan definitivamente como las mayores consumidoras de drogas legales y residuales de las ilegales, al ser comparadas con los varones.

- Si bien es cierto que la construcción social del género sigue manteniendo a las mujeres en una doble esfera: pública y privada, esta situación es similar en las distintas consumidoras de drogas. Sin embargo, la vida pública es diferente para las mujeres que usan éxtasis: su nivel educativo es alto, en muchos casos es universitario. No se han visto necesariamente relacionadas con la prostitución y sus problemas con la justicia son escasos. Este perfil se podría corresponder mejor con la consumidora de cocaína aspirada en el Perú y no con la usuaria de PBC.

- Pensamos que sería importante incorporar la perspectiva de género en la recogida de datos de países como el Perú, que no mantienen una periodicidad constante en sus estudios epidemiológicos. 
Peruanas y españolas que consumen drogas

Metodologías cualitativas y aproximación al discurso de las mujeres serían una opción para poder recoger sus prácticas y a partir de ellas mejorar las formas de intervención entre los colectivos de usuarias de drogas.

\section{Referencias}

CONTRADROGAS (1998). Encuesta nacional sobre prevención y uso de drogas. Informe general. CONTRADROGAS-INEI.

Dador, J. (1998). La violencia contra la mujer. Estudio de casos sobre la aplicación de la ley de violencia familiar, desde una perspectiva de género. Lima.

Ferrando, D. (1990). Uso de drogas en las ciudades del Perú. Encuesta de hogares-1998. Monografía de Investigación No. 5. Lima: CEDRO.

Jutkowitz, J. M., Arellano, R., Castro de la Mata, R., Davis, P. B., Elison, J., Jerí, F. R., Shaycoft, M. Y. y Timana, J. (1987). Uso y abuso de drogas en el Perú. Una investigación epidemiológica de drogas en el Perú urbano. Monografía de Investigación No. 1. Lima: CEDRO.

DGPND (Delegación del Gobierno para el Plan Nacional sobre Drogas) (1996). Encuesta Domiciliaria sobre Uso de Drogas. Madrid: Ministerio del Interior.

DGPND (Delegación del Gobierno para el Plan Nacional sobre Drogas) (1997). Encuesta sobre Drogas a la Población Escolar, 1996. Madrid: Ministerio del Interior.

DGPND (Delegación del Gobierno para el Plan Nacional sobre Drogas) (1999). Encuesta Domiciliaria sobre Uso de Drogas. Madrid: Ministerio del Interior.

Domic, Z. (1980). Revisión crítica-bibliográfica y consideraciones generales acerca del masticado de coca. En R. Jerí (Ed.), Cocaína. Actas del seminario interamericano sobre coca y cocaína (pp. 201-205). Lima: Pacific Press. 
EMCDDA (European Monitoring Centre for Drug and Drug Addiction) (1997). Annual report on the state of the drugs problem in the European Union. Lisboa: autor.

Esteban, M. L. (2001). El género como categoría analítica. Revisiones y aplicaciones a la salud. En Miqueo, C.; Tomás, C.; Tejero, C.; Barral, M.J.; Fernández, T.; y Yago, T. (eds.): Perspectivas de género en salud. Fundamentos científicos y socioprofesionales de diferencias sexuales no previstas. Minerva. Madrid, 2001.

Estébanez Estébanez, P. (1998). HIV risk behaviour in female injecting drug users in Madrid, Spain. Tesis Doctoral. London School of Hygiene and Tropical Medicine, Londres.

Gamella, J. F. y Alvarez Roldán, A. (1999). Las rutas del éxtasis. Drogas de sintesis y nuevas culturas juveniles. Barcelona: Ariel. Gamella, J. F., Alvarez Roldán, A. y Romo, N. (1997). The content of «ecstasy» in Spain. Analysis of a street sample. En D. J. Korf y H. Riper (Eds.), Illicit drugs in Europe. Amsterdam: SISWO.

Meneses, C. (2001). Mujer y heroína. Un estudio antropológico de la heroinomanía femenina. Tesis Doctoral. Departamento de Antropología y Trabajo Social-Universidad de Granada, Granada.

Molla, M. y Ragúz, M. (1997). Trastornos de identidad en mujeres con problema de drogas. Programa de la Naciones Unidas para la Fiscalización Internacional de Drogas.

Orte Socias, C. (1998). Sexualidad, mujer y drogodependencia. Revista Proyecto 26, 7-13.

Ortiz, T. (1997). Feminismos, mujeres y ciencia. En F. J. Rodríguez, R. M. Medina y J. A. Sánchez (Eds.), Ciencia, tecnología y sociedad. Contribuciones para una cultura de la paz (pp. 185-203) . Granada: Universidad de Granada.

Roig, A. (1990). Extasis: Una revisión del MDMA, MDA y demás feniletilaminas psicoactivas. XVII Jornadas Nacionales de Sociodrogalcohol. Libro de ponencias, Valencia: Diputación Provincial.

Rojas, M. (1989). Incidencias de toxicomanías. Características psicosociales y algunas observaciones clínicas en un grupo de fármaco-dependientes. Informativo de CEDRO, 15, 9-14. 
Peruanas y españolas que consumen drogas

Rojas, M. (1996). Consumo de pasta básica de cocaína en mujeres. Un estudio desde la perspectiva de género. Lima: CEDROEDEX KOLEKTIBOA.

Rojas, M. (1998). Mujeres que consumen sustancias psicoactivas. Un estudio psicológico, social y cultural sobre 627 casos. Lima: CEDRO-NAS.

Rojas, M. (1999). Asociaciones entre consumo de sustancias psicoactivas en mujeres y la conducta de prostitución. Cuadernos de Información - Serie Prevención, 4.

Rojas, M. y Castro de la Mata, R. (1997). Epidemiología de drogas en la población urbana peruana-1995. (Encuesta en hogares). Monografía de Investigación No. 15. CEDRO, Lima.

Rojas, M. y Castro de la Mata, R. (1999). Epidemiología de drogas en la población urbana peruana-1997. Monografía No. 17. CEDRO, Lima.

Rojas, M. y Ferrando, D. (1993). Drogas en el Perú urbano. Estudio epidemiológico 1992. Monografía de Investigación No. 9. Lima: CEDRO.

Romo, N. (2001a). Mujeres y drogas de síntesis. Género y riesgo en la cultura del baile. Donostia, Gakoa.

Romo, N. (2001b). Género y percepción de riesgo en los nuevos usos de "drogas de síntesis". Instituto de Estudio de las Adicciones. E-boletín. www.ieanet.com/boletín/index.htm.

Vigo, M. (1999). Creencias irracionales en varones y mujeres dependientes a la pasta básica de cocaína. Tesis para optar el título de Licenciada en Psicología, Pontificia Universidad Católica del Perú, Lima. 